University of Nebraska - Lincoln

DigitalCommons@University of Nebraska - Lincoln

2009

\title{
Two distinct waxy alleles impact the granule-bound starch synthase in sorghum
}

\author{
Scott E. Sattler \\ University of Nebraska-Lincoln, Scott.Sattler@ars.usda.gov \\ Jaswinder Singh \\ McGill University \\ Eric J. Haas \\ Creighton University, EricHaas@creighton.edu \\ Lining Guo \\ University of Nebraska - Lincoln \\ Gautam Sarath \\ University of Nebraska - Lincoln, Gautam.sarath@ars.usda.gov \\ See next page for additional authors
}

Follow this and additional works at: https://digitalcommons.unl.edu/biochemistrysarath

Part of the Biochemistry, Biophysics, and Structural Biology Commons

Sattler, Scott E.; Singh, Jaswinder; Haas, Eric J.; Guo, Lining; Sarath, Gautam; and Pedersen, Jeffrey F., "Two distinct waxy alleles impact the granule-bound starch synthase in sorghum" (2009). Gautam Sarath Publications. 14.

https://digitalcommons.unl.edu/biochemistrysarath/14

This Article is brought to you for free and open access by the Biochemistry, Department of at DigitalCommons@University of Nebraska - Lincoln. It has been accepted for inclusion in Gautam Sarath Publications by an authorized administrator of DigitalCommons@University of Nebraska - Lincoln. 


\section{Authors}

Scott E. Sattler, Jaswinder Singh, Eric J. Haas, Lining Guo, Gautam Sarath, and Jeffrey F. Pedersen 


\title{
Two distinct waxy alleles impact the granule-bound starch synthase in sorghum
}

\author{
Scott E. Sattler $\cdot$ Jaswinder Singh $\cdot$ \\ Eric J. Haas · Lining Guo · Gautam Sarath · \\ Jeffrey F. Pedersen
}

Received: 24 March 2009/Accepted: 11 May 2009/Published online: 5 June 2009

(C) US Government 2009

\begin{abstract}
The granule-bound starch synthase (GBSS) is the enzyme responsible for amylose synthesis in starch granules. Loss of GBSS activity results in starch granules containing mostly amylopectin and little or no amylose, a phenotype described as waxy. Previously, two phenotypic classes of waxy alleles were identified in sorghum (Sorghum bicolor $\mathrm{L}$. Moench) characterized by the absence $\left(w a x y^{a} ; w x^{a}\right)$ or presence $\left(w a x y^{b} ; w x^{b}\right)$ of the GBSS protein in the endosperm. To characterize these alleles, we examined endosperm architecture using scanning electron microscopy (SEM), assayed GBSS enzymatic
\end{abstract}

S. E. Sattler $(\bowtie) \cdot$ L. Guo · G. Sarath · J. F. Pedersen Grain, Forage and Bioenergy Research Unit, USDA-ARS, University of Nebraska-Lincoln, Lincoln,

NE 68583-0739, USA

e-mail: Scott.Sattler@ars.usda.gov

S. E. Sattler · L. Guo · G. Sarath · J. F. Pedersen Department of Agronomy and Horticulture, University of Nebraska-Lincoln, Lincoln, NE 68583-0739, USA

\section{J. Singh}

Department of Plant Science, McGill University, Ste.

Anne de Bellevue, Montreal, QC H9X 3V9, Canada

\section{E. J. Haas}

Department of Chemistry, Creighton University, 2500 California Plaza, Omaha, NE 68178, USA

Present Address:

L. Guo

Metabolon Inc., Durham, NC 27713, USA activities, and identified DNA lesions associated with the mutations in the GBSS (Sb10g002140) gene. $w x^{a}$, the allele present in B Tx630 and R Tx2907, contained a large insertion in the third exon, which was consistent with the absence of the GBSS protein previously observed. $w x^{b}$, the allele present in B 9307 and B TxARG1, contained a missense mutation that resulted in conversion of glutamine 268 to histidine in a conserved domain in starch synthases. In $w x^{b}$, GBSS activity was less than $25 \%$ that of the non-waxy line B Wheatland, and GBSS activity was not detected in $w x^{a}$. SEM showed that endosperm architecture was very similar in both $w x^{a}$ and $w x^{b}$ alleles, but altered in comparison to non-waxy lines R Tx430 and B Wheatland. Both alleles may have a range of potential applications in grain sorghum because of low amylose content in their starch and the presence or absence of the GBSS protein. PCR based markers were developed for both the $w x^{a}$ and the $w x^{b}$ alleles to aid in molecular breeding of low amylose sorghum.

Keywords Waxy · Granule-bound starch synthase . Sorghum bicolor $\cdot$ Amylose $\cdot$ Amylopectin

\section{Introduction}

Starch is a major energy source for the dietary requirements of both humans and livestock. In addition, starch is also an essential substrate for 
biofuel production because it is the primary carbon source for ethanol production in the United States. In photosynthetic tissues, starch is synthesized in the chloroplast during the day from the photosynthetically fixed carbon and is catabolized to glucose during the night when photosynthetically fixed carbon is unavailable (Zeeman et al. 2007). More importantly for agriculture, some plants also accumulate large quantities of starch in non-photosynthetic storage tissues such as tubers, stems, roots and seeds. In grasses, seeds contain a large, well-developed endosperm, which is filled with tightly packed starch granules, the main carbon source for the germinating seed.

Starch consists of two types of glucose polymers: the almost entirely linear form amylose (linear molecule of $\alpha-1,4$ linked glucose monomers) and the highly branched form amylopectin $(\alpha-1,4$ glucan with $\alpha-1,6$ branch points) (James et al. 2003; Zeeman et al. 2007). In different plant species, the amylose content in storage tissues ranges from 11 to $37 \%$ of the starch (Nakamura et al. 1995). There are five classes of starch synthases, which catalyze the addition of glucose molecules from ADP-glucose to the nonreducing end of the polymer creating an $\alpha-1,4$ linkage (James et al. 2003). However, the granulebound starch synthase (GBSS) is the sole enzyme responsible for amylose biosynthesis in cereal endosperm (Denyer et al. 2001). This $60 \mathrm{kD}$ protein is almost entirely localized to starch granules, where it is enzymatically active both at the periphery and in the interior of the starch granule, unlike the other starch synthase isoforms (Denyer et al. 2001). Loss of GBSS function in planta causes the waxy endosperm phenotype, named for its altered texture and appearance of the endosperm (Denyer et al. 2001). Waxy mutants contain very low or undetectable levels of amylose, and their starch granules are nearly entirely composed of amylopectin (Denyer et al. 2001). Waxy mutants affecting either gene expression or function of GBSS have been isolated in several different plants including: pea (Pisum sativum) (Denyer et al. 1996), wheat (Triticum aestivum) (Nakamura et al. 1995), rice (Oryza sativa) (Isshiki et al. 1998; Larkin and Park 1999; Wang et al. 1995), barley (Hordeum vulgare) (Hylton et al. 1996; Taira et al. 1995) and corn (Zea mays) (Shure et al. 1983). In sorghum, two classes of waxy mutants were previously identified based on the near absence of amylose and were classified based on absence $\left(\right.$ waxy $\left.^{a}\right)$ or presence $\left(w_{a x y}^{b}\right)$ of the GBSS protein (Pedersen et al. 2005).

Because the waxy mutation alters starch composition by greatly reducing amylose content, the physiochemical properties of waxy starch are altered relative to normal starch (Pedersen et al. 2007; Sang et al. 2008), which has some potential applications for sorghum. Recently, waxy sorghum grain was demonstrated to have higher ethanol conversion rates than wild-type grain (Wang et al. 2008), which may make waxy loci useful in bioenergy grain sorghum. In addition, waxy grain has been shown to increase starch and protein digestibility compared to wild-type grain (Rooney and Pflugfelder 1986; Wong et al. 2009).

Here, we have identified DNA lesions and characterized the biochemical mechanisms of sorghum waxy lines; B Tx630, R Tx2907, B 9307 and B TxARG1. This information gives us new tools to study altered sorghum starch composition and its impact on grain traits.

\section{Materials and methods}

Plant material and growth conditions

Sorghum lines B 9307, B Tx630, B TxARG1, B Wheatland, R Tx2907 and R Tx430 were grown in experimental testing fields in Lincoln, Nebraska and harvested for this study. For biochemical analysis and gene cloning, developing seeds at 14 weeks after flowering (soft dough stage) were harvested, immediately frozen in liquid nitrogen, and stored at $-80^{\circ} \mathrm{C}$.

Extraction and purification of starch

About $5 \mathrm{~g}$ of seeds was homogenized in a blender with $40 \mathrm{ml}$ extraction buffer (100 mM Tris, $\mathrm{pH} \mathrm{7.2,}$ $2 \mathrm{mM}$ EDTA and $1 \mathrm{mM}$ DTT). The extract was filtered through four layers of cheesecloth and centrifuged at $905 \mathrm{~g}$ at $4^{\circ} \mathrm{C}$ for $5 \mathrm{~min}$. The pellet was suspended in $15 \mathrm{ml}$ extraction buffer, layered on $15 \mathrm{ml} 80 \% \mathrm{CsCl}$ and centrifuged at $16,000 \mathrm{~g}$ at $4^{\circ} \mathrm{C}$ for $4 \mathrm{~min}$. The starch pellet was washed twice with extraction buffer and stored in $1 \mathrm{ml}$ of extraction buffer with $50 \%$ glycerol at $-20^{\circ} \mathrm{C}$. 
Granule-bound starch synthase assay

The GBSS activity was assayed using the method previously described in (Tenorio et al. 2003). Starch granules were washed three times in $100 \mathrm{mM}$ Tris buffer, $\mathrm{pH}$ 7.2, $2 \mathrm{mM}$ EDTA and $1 \mathrm{mM}$ DTT. $5 \mathrm{mg}$ starch was suspended in $100 \mu \mathrm{l}$ of $100 \mathrm{mM}$ Tris, $\mathrm{pH}$ 7.2, $10 \mathrm{mM}$ DTT, $5 \mathrm{mM}$ EDTA, and $1 \mathrm{mM}$ ADP[U$14 \mathrm{C}$ ]-glucose (specific activity $7 \mathrm{GBq} / \mathrm{mol}$ ). The reaction mixture was incubated at room temperature $\left(25^{\circ} \mathrm{C}\right)$ for $15 \mathrm{~min}$ with continuous rotation to prevent precipitation of starch granules. At the end of incubation, $1 \mathrm{ml}$ solution of $70 \%$ methanol, $1 \%$ $\mathrm{KCl}$ was added to wash the starch. The washing step was repeated two additional times. The radioactivity incorporated into the starch granules was then quantified using a scintillation counter.

\section{Cloning and sequencing of GBSS $w x^{a}$}

High-efficiency thermal asymmetric interlaced PCR (hiTAIL-PCR) was performed as described (Liu and Chen 2007) to amplify the $5^{\prime}$ unknown region of the waxy $a$ allele, which failed PCR amplification with a primer pair designed to amplify the second and third exons. The following are the two sets of primers specific to the 4th exon of Sb10g002140.1: Wxy-0a $5^{\prime}$-tgctaccaatgggtgcctattatcatgttcagc- $3^{\prime}, \mathrm{Wxy}-0 \mathrm{~b} 5^{\prime}$-ca aacctgaaaccagcgaccagacgaag- $3^{\prime}$, Wxy-1 as $5^{\prime}$-acgatgg actccagtccggcctctcaaggaacagcgggtggtcaatg- $3^{\prime}$, Wxy-1bs $5^{\prime}$-acgatggactccagtccggccggaatctcaccetctcaaggaacagcgg$3^{\prime}$, Wxy-2a $5^{\prime}$-aaaaacctcaccgtctcgtacccgtctcc- $3^{\prime}$, Wxy-2b $5^{\prime}$-tggaaaaacctcaccgtctcgtacccg-3'. The resulting PCR products were agarose gel purified and cloned into pCR-Blunt vector (Invitrogen; Carlsbad, CA). The Genomic Core Research Facility at the University of Nebraska-Lincoln performed automated DNA sequencing of cloned PCR products using M13 and T7 primers for the polylinker of the vector.

A set of three PCR primers was designed using MacVector (Cary, NC) to amplify both the $w x$ and wild-type alleles using the same reverse primer Wx-R $5^{\prime}$-gcagctggttgtccttgtag- $3^{\prime}$. wx-a-F $5^{\prime}$-cgtggcgagatcaaactcta- $3^{\prime}$ amplified a 615 bp fragment containing the junction between $w x^{a}$ insertion and exon 5 . Wx-F $5^{\prime}$-ggcctggattcaatgttctt- $3^{\prime}$ amplified a 523 bp fragment containing portions of intron 2 and exon 5 . The paired primers were tested separately using both wild-type and $w x^{a}$ templates. The three primers were combined in a single PCR reaction to detect wildtype, $w x^{a}$ or heterozygous plants.

Cloning and sequencing of GBSS $w x^{b}$

Approximately twenty seeds were harvested, and pericarp and embryo were removed. The endosperm was then frozen in liquid nitrogen and stored at $80^{\circ} \mathrm{C}$. Total RNA was extracted using Trizol reagent (Invitrogen, Carlsbad, CA) according to the manufacturer's instructions. RNA was further cleaned and purified using RNeasy Mini Kit (Qiagen, Valencia, CA) according to the manufacturer's instructions.

cDNA was synthesized in a reverse transcription (RT) reaction from $1 \mu \mathrm{g}$ total RNA and a reverse primer (5'-ccgctcgagtcagggtgcggccacgttctcc- $3^{\prime}$ ) designed for sorghum GBSS gene using SuperScript III First-Strand Synthesis System (Invitrogen). PCR was then carried out in a total volume of $50 \mu \mathrm{l}$ using $2 \mu \mathrm{l}$ of the above RT reaction mixture and primers $\left(5^{\prime}\right.$-ccgctcgagtcagggtgc ggccacgttctcc- $3^{\prime}$ and $5^{\prime}$-atggtaccatgtcgactctagccacgtcg$3^{\prime}$ ) with AccuPrime pfx DNA polymerase (Invitrogen) according to the manufacturer's instructions. The resulting PCR (Liu and Chen 2007) product was digested with restriction endonucleases $\mathrm{XhoI}$ and $\mathrm{KpnI}$ (New England Biolabs, Ipswich, MA), cloned into pET30a (+) vector (Novagen, Madison, WI), and sequenced. For the Cleavage Amplified Polymorphic Sequence (CAPS) marker for $W x$, (forward $5^{\prime}$-cgac cgtgtgttcattgaccac- $3^{\prime}$ reverse $5^{\prime}$-ttgttcagtgcctttgcctcg$3^{\prime}, 2 \mu \mathrm{l}$ of $20 \mu \mathrm{l}$ PCR reaction were digested with 2.5 units of NcoI and analyzed by agarose gel electrophoresis. $w x^{b}$ PCR product contained a single NcoI restriction site following the digest, generating $745 \mathrm{bp}$ and $537 \mathrm{bp}$ DNA fragments. The wild-type PCR product did not contain a $N c o$ I restriction site and a single $1281 \mathrm{bp}$ fragment was observed.

\section{Protein modeling}

A model of Sorghum bicolor GBSS was created using SWISS-MODEL (Arnold et al. 2006; Kopp and Schwede 2004, 2006; Peitsch 1996; Schwede et al. 2003) and the protein database (Berman et al. 2000). The structure of closed form Escherichia coli glycogen synthase (PDB id 2qzs) (Sheng et al. 2009) was used as a template for model construction. The conserved regions of sequence (see Fig. 2) were forced into alignment during the modeling phase. 
Scanning electron microscopy

Several mature seeds from Wheatland, B Tx630, B 9307, R Tx430, R Tx 2907, B TxARG1 were handsectioned using a razor blade. The microscopic analyses was conducted using a Hitachi TM 1000 environmental scanning electron microscope (Hitachi High Technologies America, Inc., Pleasanton, CA) as described by Wong et al. (2009). Observations were made at $60 \times, 250 \times, 1000 \times$ and $5000 \times$ magnifications of the endosperm.

\section{Results}

Identification of the waxy $^{a}$ and $w^{2} y^{b}$ alleles

The GBSS gene (Waxy) was identified in sorghum based on sequence similarity to other known Waxy genes. Based on the Sorghum Genome Project, the sorghum Wx gene was identified as Sb10g002140.1, which contained 14 exons (Fig. 1a) and encoded a protein containing 609 amino acids. To determine the mutations responsible for the waxy phenotype, the $W x$ cDNA was amplified using RT-PCR from R Tx430 (non-waxy), B Wheatland (non-waxy), B Tx630 $\left(w x^{a}\right), \mathrm{R}$ Tx2907 $\left(w x^{a}\right)$, B $9307\left(w x^{b}\right)$ and B TxARG1 $\left(w x^{b}\right)$. The RT-PCR failed to amplify cDNA for either B Tx630 $\left(w x^{a}\right)$ or $\mathrm{R}$ Tx2907 $\left(w x^{a}\right)$. DNA sequencing of B $9307\left(w x^{b}\right)$ and B TxARG1 $\left(w x^{b}\right)$ cDNA showed that a $\mathrm{C}$ to $\mathrm{T}$ transition caused a missense mutation changing amino acid 268 from glutamine to histidine (Fig. 1a). This result is consistent with two earlier publications, which identified a $\mathrm{C}$ to $\mathrm{T}$ transition resulting in changing amino acid 268 from a glutamine to histidine (Hamblin et al. 2007; McIntyre et al. 2008). To explore the potential impact of the conversion of amino acid 268 from a glutamine to a histidine in $w x^{b}$, the predicted amino acid sequences of glucan synthase from higher plants, green algae and eubacteria were obtained from the public database and aligned using ClustalW (Fig. 2). This dataset included other GBSS sequences in addition to plant soluble starch synthases and bacterial glycogen synthases. The glutamine 268 was conserved across all glucan synthases examined indicating that the glutamine residue may have a functional or structural role in glucan synthases. Glutamine 268 is contained within the starch synthase catalytic domain, but outside of the putative active site amino acids (96-100) (Denyer et al. 2001). A structural model of sorghum GBSS protein was constructed using Swiss Model and the crystal structure of the E. coli glycogen synthase as the template (Fig. 3). Gln 268 lies $\sim 6 \AA$ away from the substrate binding pocket. Unfortunately, the model did not provide insight into how the amino acid change from the polar side chain of glutamine to the positively charged side chain of histidine might affect $w x^{b}$ protein structure or function.

A Cleavage Amplified Polymorphic Sequence (CAPS) marker was designed to detect this mutation. $w x^{b}$ mutation created a NcoI restriction site, which was absent in wild-type and $w x^{a}$ alleles. NcoI restriction digest of the $1281 \mathrm{bp}$ PCR product resulted in cleavage of the $w x^{b}$ product into $745 \mathrm{bp}$ and 537 bp DNA fragments, while wild-type and $w x^{a}$ PCR amplified products remained uncleaved (Fig. 1c). The CAPS marker confirmed that both B 9307 and B TxARG1 contained the same missense mutation in GBSS, which is consistent with B 9307 and B TxARG1 sharing a common pedigree.

The PCR results suggested that the $w x^{a}$ mutation affected the transcription the GBSS gene. PCR primers were designed to amplify the genomic coding region of the Waxy locus. The primer pair designed to amplify the genomic region containing exons 1-4 failed to amplify a PCR product in $w x^{a}$, a region that was amplified by PCR in wild-type (non-waxy). Sequences corresponding to these primers were present in other amplification products from this region, suggesting that a large insertion in $w x^{a}$ might be the reason the primers failed to amplify a product. To amplify the unknown region upstream of the 4th exon from $w x^{a}$, high-efficiency Thermal Asymmetric InterLaced PCR (hiTAIL-PCR) (see Materials and methods) was used together with reverse primers that complemented the sequence from the 4th exon, which was amplified from $w x^{a}$. The hiTAIL-PCR amplified products, which ranged in size from 1.5 to $4 \mathrm{~kb}$, were cloned into a plasmid vector and sequenced. DNA sequencing revealed that the GBSS locus in $w x^{a}$ contained an insertion within the 3rd exon. The largest hiTAIL-PCR product cloned was $\sim 4 \mathrm{~kb}$ in size, and the $5^{\prime}$ end of the insert did not contain sequences corresponding to GBSS locus. The DNA sequence from the insertion was analyzed using RepeatMasker (http://www.repeatmasker.org), which 
A
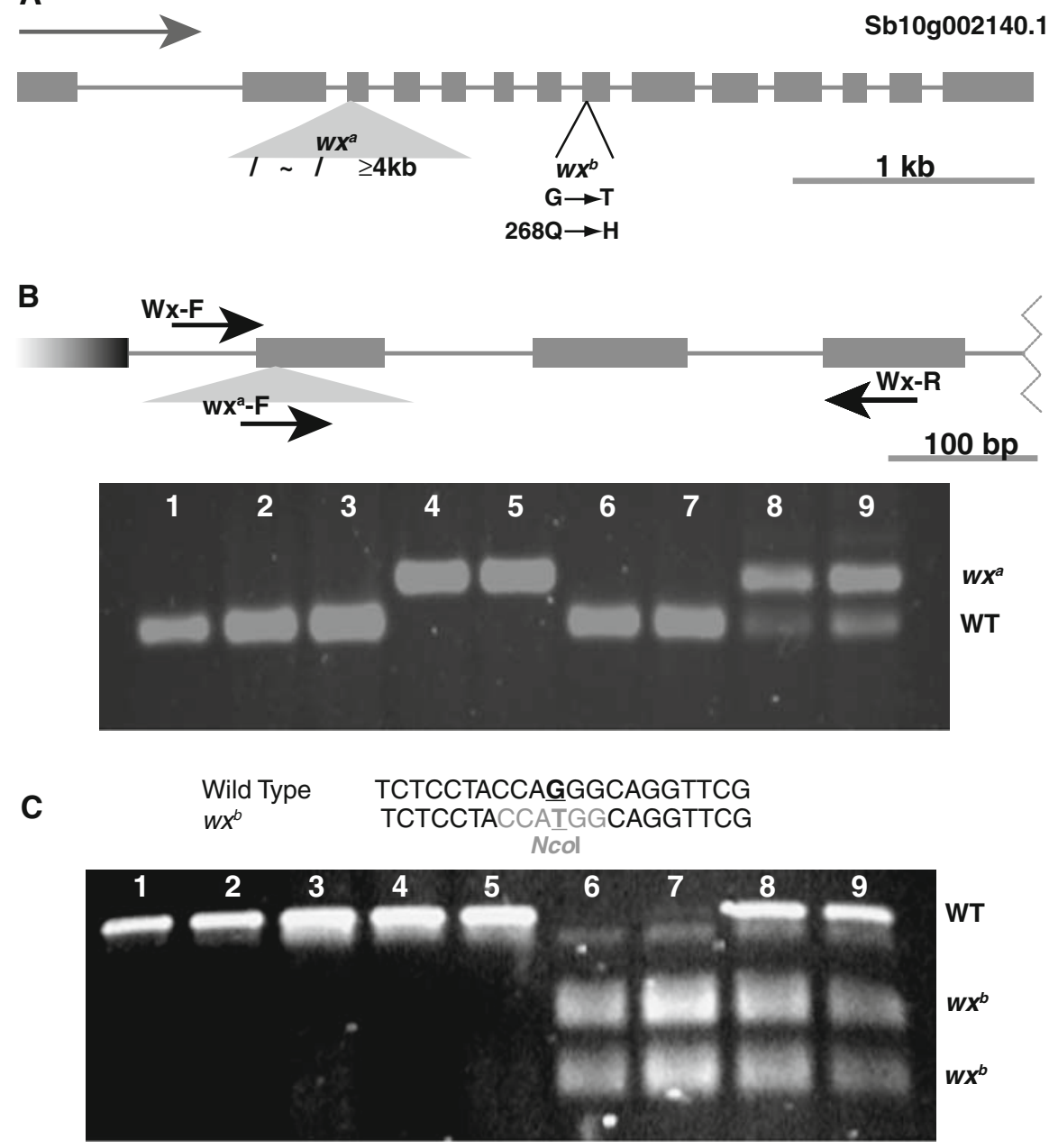

Fig. 1 Waxy locus and $w x^{a}$ and $w x^{b}$ mutations. a The sorghum granule bound starch synthase (Waxy) locus (Sb10g002140.1) is depicted; the exons are represented as rectangles and the introns as lines. The position of the insertion in $w x^{a}$ and the missense mutation in $w x^{b}$ are also shown. b A set of three PCR primers was designed to amplify a 523 bp band specific to wild-type and a 615 bp band specific $w x^{a}$ in order to determine the presence or absence of the $w x^{a}$ insert. 1 Wheatland (wildtype), 2 R Tx430 (wild-type), 3 Atlas (wild-type), 4 B Tx630 $\left(w x^{a}\right), 5$ R Tx $2907\left(w x^{a}\right), 6$ B $9307\left(w x^{b}\right), 7$ B TxARG1 $\left(w x^{b}\right)$, 8 Synthetic heterozygote (B Tx630/R Tx430), 9 Synthetic

showed that the insertion contained sequences that were similar to Ty1/Copia retroelements and En-Spm transposons. Based on the hiTAIL-PCR results, $w x^{a}$ contained an insertion of repetitive DNA that is greater than $4 \mathrm{~kb}$ in size. The $w x^{a}$ insertion is located just up-stream of the DNA sequence encoding amino acid 116. This insertion separates the majority of the heterozygote (R Tx2907/R Tx430). c A CAPS (Cleavage Amplified Polymorphic Sequence) marker was designed to detect the presence or absence of the $w x^{b}$ missense mutation. $w x^{b}$ mutation creates a NcoI restriction site that is absent in wild-type. The PCR product from $w x^{b}$ was cleaved by NcoI resulting in 745 bp and 537 bp DNA fragments, and wild-type $1,281 \mathrm{bp}$ PCR product remained intact. 1 Wheatland (wildtype), 2 R Tx430 (wild-type), 3 Atlas (wild-type), 4 B Tx630 $\left(w x^{a}\right), 5$ R Tx $2907\left(w x^{a}\right), 6$ B $9307\left(w x^{b}\right), 7$ B TxARG1 $\left(w x^{b}\right)$, 8 Synthetic heterozygote (B 9307/R Tx430), 9 Synthetic heterozygote (B TxAgri1/R Tx430)

coding region from the promoter, $5^{\prime}$ UTR, chloroplast transit peptide and the highly conserved cereal motif surrounding the putative active site of the enzyme (Fig. 1a) (Baldwin 2001; Denyer et al. 2001; Taira et al. 1995). This result is consistent with the absence of GBSS protein previously observed (Pedersen et al. 2005) and the inability to amplify GBSS transcript. 


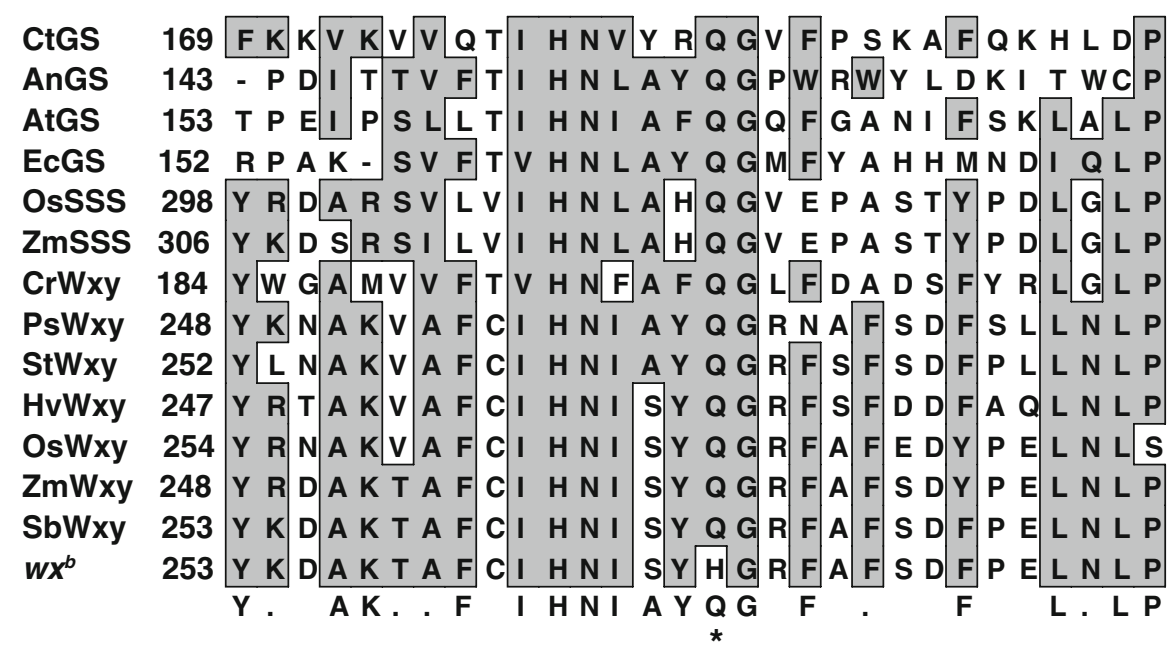

Fig. 2 Amino acid sequence alignment of glycan synthases. Predicted amino acid sequences for glycan synthases from a range of organisms were aligned using ClustalW. Bacterial Glycogen Synthases from Chlorobium tepidum CtGS (Q8KAY6); Anabaena sp. strain PCC 7120 AnGS (Q8YVU5); Agrobacterium tumefaciens AtGS (P0A3F2 P39670) and Escherichia coli EcGS (P0A6V0 P08323). Soluble starch synthases from rice OsSSS (BAA07396) and maize ZmSSS

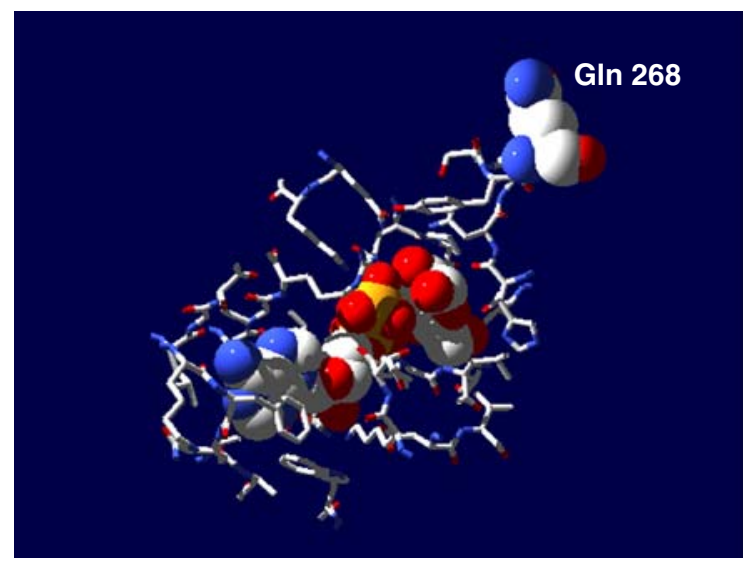

Fig. 3 A view of Gln 268 in relation to the substrate binding groove for a model of GBSS. The bound substrates, ADP and glucose, are shown in space-filling (CPK; Corey, Pauling and Koltun) representation. Surrounding amino acids are included. Gln 268, 6 8 distant from the substrates, is also shown in CPK representation near the upper right corner of the diagram. This figure was created with the DeepView molecular viewing program (Guex and Peitsch 1997) and rendered using POVRay ray-tracing program (http://www.povray.org)

A PCR-based marker derived from a set of three PCR primers was designed to identify the $w x^{a}$ and wild-type alleles of GBSS (Fig. 1b). A 615 bp
(CAB69545). Granule bound starch synthases from Chlamydomonas reinhardtii CrWxy (EDP08594); pea PsWxy (CAA 61268); potato StWxy (Q00775 Q43176); barley HvWxy (CAA30756); rice OsWxy (CAA37732); maize ZmWxy (CAA27574); sorghum SbWxy (Q43134 P81888) and $w x^{b}$ (this article). The alignment includes the amino acid sequence surrounding the sorghum $w x^{b}$ missense mutation, which is denoted by an *. Gray shading denotes conserved amino acids

fragment containing the $w x^{a}$ insertion to the 5th exon was amplified in $w x^{a}$ and a $523 \mathrm{bp}$ fragment containing portions from the 2 nd intron to the 5 th exon was amplified in wild-type. The $w x^{a}$ marker confirmed that B Tx630 and R Tx 2907 contained the same insertion in the GBSS gene. These PCR-based markers for $w x^{a}$ and $w x^{b}$ should be useful for future breeding efforts using the waxy phenotype, because plants can be screened at very early stages and these markers are co-dominant, so heterozygous plants can be identified (Fig. 1b and c).

\section{GBSS activity in isolated starch granules}

To determine the effects of the $w x^{a}$ and $w x^{b}$ mutations on GBSS activity, starch granules were isolated from developing seeds at 14 weeks after flowering (soft dough stage) and washed to remove soluble starch synthases. The incorporation of radiolabeled ADP- $\left[{ }^{14} \mathrm{C}\right]$ glucose was used to measure GBSS activity in the washed starch granules from B Wheatland (wild-type), R Tx2907 ( $\left.w x^{a}\right)$, B 9307 $\left(w x^{b}\right)$ and B TxARG1 $\left(w x^{b}\right)$. Corresponding tissue was not available for R Tx430 (wild-type) and B 


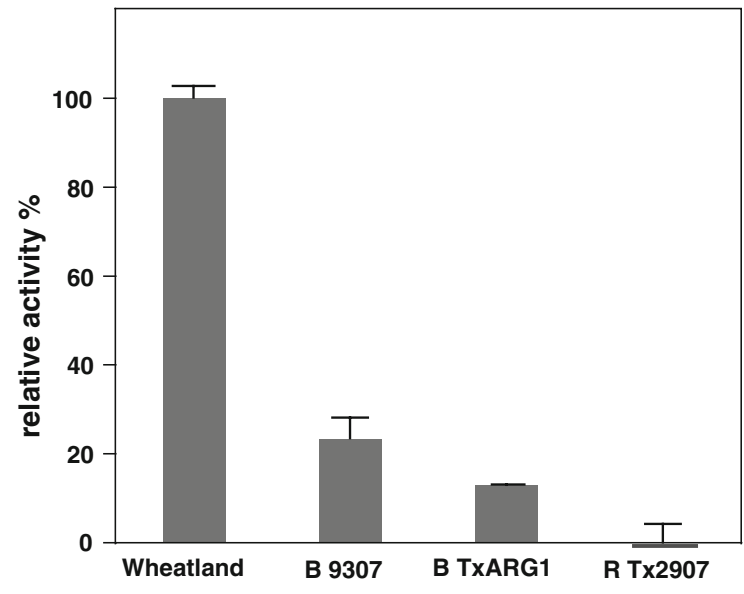

Fig. 4 Granule-bound starch synthase activity. GBSS activity was quantified in the starch granules isolated from developing endosperm of Wheatland (WT), B $9307\left(w x^{b}\right)$, TxARG1 $\left(w x^{b}\right)$ and Tx2907 $\left(w x^{a}\right)$ plants based on incorporation of ${ }^{14} \mathrm{C}$ labeled ADP-glucose. The activity was expressed relative to Wheatland (WT). This experiment was repeated twice with similar results. $n=3$, error bars $=$ standard deviation

Tx630 ( $\left.w x^{a}\right)$ when the assays were performed. Isolated starch granules from both B $9307\left(w x^{b}\right)$ and B TxARG1 $\left(w x^{b}\right)$ incorporated the radiolabeled glucose at 23 and $13 \%$ of wild-type (Wheatland) incorporation levels, respectively (Fig. 4). However, the difference in radiolabeled glucose incorporation between B $9307\left(w x^{b}\right)$ and B TxARG1 $\left(w x^{b}\right)$ was not statistically significant based on Student's t-test. Incorporation of the radiolabeled glucose in $\mathrm{R}$ Tx2907 $\left(w x^{a}\right)$ was not detected after the $15 \mathrm{~min}$ incubation period. Both $w x$ alleles resulted in significantly reduced GBSS activity, which is consistent with previous results.

\section{Scanning electron microscopy}

To examine the effect of waxy alleles on the sorghum kernel, mature kernels were sectioned and analyzed using scanning electron microscopy. Distinct differences between the endosperm of wild-type and $w x$ kernels were apparent even under low magnification $(60 \times)$ (Fig. 5). All $w x$ lines had smaller floury endosperm (F) compared to wild-type B Wheatland and $\mathrm{R} T \mathrm{~T} 430$, and most of the floury region surrounded the embryo in contrast to wild-type where the floury region extended into the center of the grain (Fig. 5, top panel). The less compact corneous (LC) was larger and found in the middle of the seed in all waxy lines, whereas it was observed surrounding the floury endosperm in wild-type.

The grain from R Tx2907 $\left(w x^{a}\right)$ and B Tx630 $\left(w x^{a}\right)$ were nearly indistinguishable from each other as was the grain from B TxARG1 $\left(w x^{b}\right)$ and B 9307 $\left(w x^{b}\right)$ (Fig. 5). At higher magnification $(1000 \times)$, the corneous endosperm (C) was more compact in wildtype than in $w x^{a}$ or $w x^{b}$ (Fig. 5, second panel). LC endosperm was more loosely packed in the $w x$ kernels compared to wild-type, and $w x^{a}$ LC region was slightly more loosely packed than $w x^{b}$ (Fig. 5, third panel). However, the $w x^{a}$ starch granules in the LC region were larger and more similar to wild-type than $w x^{b}$. The starch granules of the floury endosperms were slightly smaller in $w x^{b}$ than in wild-type or $w x^{a}$ (Fig. 5, bottom panel). Overall, the wild-type grain appeared to be more tightly packed than either $w x$ allele regardless of variety. Both $w x$ alleles were more similar to each other than to wild-type.

\section{Discussion}

GBSS is the sole enzyme responsible for synthesizing amylose starch polymer from ADP-glucose, which only occurs inside starch granules. The mutations in $w x^{a}$ and $w x^{b}$ alleles, respectively, have been identified in the GBSS gene in this article $\left(w x^{a}\right.$ and $\left.w x^{b}\right)$ and previously $\left(w x^{b}\right)$ (Hamblin et al. 2007; McIntyre et al. 2008). The insertion in $w x^{a}$ separates the promoter and first two exons from the rest of the coding region, which is predicted to result in a non-functional gene product, consistent with the previous finding that the GBSS protein was absent from $w x^{a}$ seeds (Pedersen et al. 2005). The $w x^{b}$ mutation is a missense mutation within a conserved domain found in both starch synthase and bacterial glycogen synthases, resulting in the conversion of glutamine 268 to histidine. Interestingly, relatively few missense mutations have been reported in GBSS, with the exception of maize (Han et al. 2002a, b) and wheat (Yanagisawa et al. 2001) compared to numerous splice site mutations, deletions and insertions. While the presence of histidine at 268 in $w x^{b}$ affects enzymatic activity, it does not affect localization of the protein to starch granules (Pedersen et al. 2005).

Both $w x^{a}$ and $w x^{b}$ alleles alter endosperm architecture compared to wild-type (non-waxy) regardless of genetic background, resulting in a reduced floury 
Fig. 5 Scanning electron micrographs from mature sorghum grains. Mature sorghum grain was dissected, frozen in liquid nitrogen and observed using a scanning electron microscope equipped with a cold stage. a Wheatland (wild-type), b B Tx630 $\left(w x^{a}\right), \mathbf{c}$ B $9307\left(w x^{b}\right), \mathbf{d ~ R}$ Tx430 (wild-type), e R Tx $2907\left(w x^{a}\right)$, f B TxARG1 $\left(w x^{b}\right)$. Top panel is an overview of the endosperm magnified $60 \times$. The second panel is the compact corneous endosperm (C) magnified $1000 \times$. The third panel is the less compact corneous endosperm (LC) magnified $1000 \times$. The bottom panel is the floury endosperm $(F)$ magnified $1000 \times$. White bar represents $100 \mu \mathrm{m}$ for the lower three panels magnified $1000 \times$
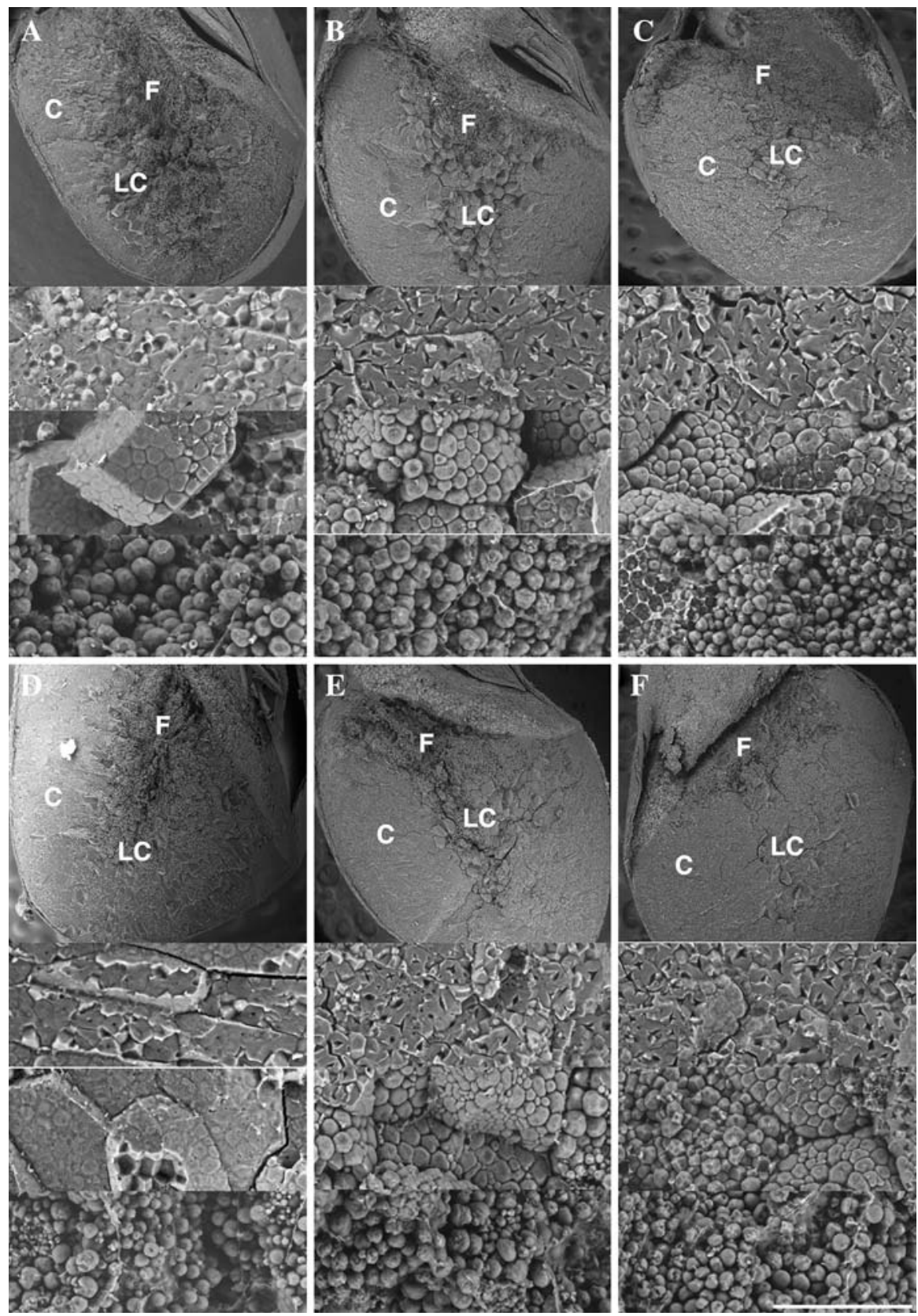

endosperm region and less densely packed starch granules in both corneous and less corneous endosperm regions compared to wild-type. More loosely packed starch granules were also observed in rice waxy mutants (Kang et al. 2006), and it is likely that these structural changes contribute to the waxy appearance of the endosperm. The $w x^{b}$ starch granules appear to be slightly smaller than those of the $w x^{a}$ or wild-type lines examined. This subtle difference in starch granule size might be related the presence of an inactive GBSS protein. The less densely packed endosperm and altered starch granules might account for the significant reductions in grain yield observed for both B Tx630 (2.7\%; wx $\left.x^{a}\right)$ and B $9307\left(8.1 \% ; w x^{b}\right)$ inbred lines relative to $\mathrm{B}$ Wheatland over 2 years (Pedersen and Toy, Unpublished).

Interestingly, $w x^{b}$ isolated starch granules were still able to incorporate radiolabeled glucose from 
ADP- $\left[{ }^{14} \mathrm{C}\right]$ glucose, but incorporation of radiolabeled glucose was negligible in $w x^{a}$ starch granules. In spite of these apparent difference of GBSS activity, both $w x^{a}$ and $w x^{b}$ starch contained only trace amounts of amylose: $1.5 \%$ B Tx630 $\left(w x^{a}\right), 1.3 \% \mathrm{R}$ Tx2907 $\left(w x^{a}\right)$, $1.8 \%$ B $9307\left(w x^{b}\right)$ and $1.1 \%$ B TxARG1 $\left(w x^{b}\right)$ compared to $23.5 \% \mathrm{R}$ Tx430 (non-waxy) and $23.0 \%$ B Wheatland (non-waxy) amylose (Pedersen et al. 2005). These data suggest that GBSS activity is still present in $w x^{b}$ isolated starch grains, but substantially lower than the activity observed in wild-type. The reasons for the discrepancy between GBSS activity and near absence of amylose in $w x^{b}$ grain are not clear at present. There is evidence that the substrate ADP-glucose or its diffusion into the starch granule limits starch synthesis in vivo (Clarke et al. 1999). Alternatively, the $w x^{b}$ GBSS protein still might be capable of synthesizing amylopectin within starch granules, but not amylose. It has been previously shown that GBSS has the ability to synthesize or extend both types of starch polymers (Delrue et al. 1992; Denyer et al. 1996; Ral et al. 2006). In maize, GBSS activity in isolated starch granules did not strongly correlate with amylose content (Tsai 1974), which suggests that there are other factors that influence amylose content in starch granules in addition to GBSS.

Both $w x^{a}$ and $w x^{b}$ derived starches have dramatically reduced amylose levels, which has been associated with altered physiochemical properties compared to normal sorghum starch (Pedersen et al. 2007). Generally, waxy starches have been shown to have low levels of retrogradation, which has some application in the food industry (Jane et al. 1999). The presence or absence of even a non-functional GBSS protein has been show to affect physiochemical properties of the starch (Han et al. 2002a, b; Pedersen et al. 2007), so there may be some potential applications specifically suited for $w x^{a}$ or $w x^{b}$ starch. Perhaps more importantly for sorghum, waxy phenotype increases starch and protein digestibility, which is a significant issue in sorghum grain fed to both humans and animals (Rooney and Pflugfelder 1986; Wong et al. 2009). This increased digestibility may be associated with reduced kernel density and the increased amount of space between starch granules observed in both $w x^{a}$ and $w x^{b}$ (Fig. 5). Both may have potential application in sorghum, even though there have been significant reductions in grain yield associated with either allele compared to non-waxy inbred lines. During fermentation of grain, amylose has been shown to form complexes with lipids that are resistant to enzymatic hydrolysis, and because $w x$ grain lacks amylose, increased ethanol conversion efficiency has been observed using $w x$ maize (Sharma et al. 2007) and sorghum (Wang et al. 2008). The yield drag associated with $w x$ remains the major barrier for its use in these applications. However, there are some indications that yield drag associated with $w x$ may be overcome through heterosis, different inbred backgrounds or a combination of these strategies (Rooney et al. 2005). PCR based markers for the $w x^{a}$ and the $w x^{b}$ alleles (this article) may aid in molecular breeding efforts to overcome the yield drag associated with low amylose sorghum.

Acknowledgments We would like to acknowledge Dr. Peggy Lemaux, Patrick O'Neill and John Toy for their technical assistance on experiments presented in this manuscript, and Drs. Robert Graybosch and Heather Van Buskirk for critically reviewing this manuscript. Mention of trade names or commercial products in this publication is solely for the purpose of providing specific information and does not imply recommendation or endorsement by the US Department of Agriculture.

\section{References}

Arnold K, Bordoli L, Kopp J, Schwede T (2006) The SWISSMODEL workspace: a web-based environment for protein structure homology modelling. Bioinformatics 22:195-201. doi:10.1093/bioinformatics/bti770

Baldwin PM (2001) Starch granule-associated proteins and polypeptides: a review. Starch-Starke 53:475-503. doi: 10.1002/1521-379X(200110)53:10<475::AID-STAR475> 3.0.CO;2-E

Berman HM, Westbrook J, Feng Z, Gilliland G, Bhat TN, Weissig H, Shindyalov IN, Bourne PE (2000) The protein data bank. Nucleic Acids Res 28:235-242. doi:10.1093/nar/ 28.1.235

Clarke BR, Denyer K, Jenner CF, Smith AM (1999) The relationship between the rate of starch synthesis, the adenosine 5 -diphosphoglucose concentration and the amylose content of starch in developing pea embryos. Planta 209:324329. doi:10.1007/s004250050639

Delrue B, Fontaine T, Routier F, Decq A, Wieruszeski JM, Van den Koornhuyse N, Maddelein ML, Fournet B, Ball S (1992) Waxy Chlamydomonas reinhardtii: monocellular algal mutants defective in amylose biosynthesis and granulebound starch synthase activity accumulate a structurally modified amylopectin. J Bacteriol 174:3612-3620

Denyer K, Clarke B, Hylton C, Tatge H, Smith AM (1996) The elongation of amylose and amylopectin chains in isolated 
starch granules. Plant J 10:1135-1143. doi:10.1046/ j.1365-313X.1996.10061135.x

Denyer K, Johnson P, Zeeman S, Smith AM (2001) The control of amylose synthesis. J Plant Physiol 158:479-487. doi:10.1078/0176-1617-00360

Guex N, Peitsch MC (1997) SWISS-MODEL and the SwissPdbViewer: an environment for comparative protein modeling. Electrophoresis 18:2714-2723. doi:10.1002/elps.11 50181505

Hamblin MT, Salas Fernandez MG, Tuinstra MR, Rooney WL, Kresovich S (2007) Sequence variation at candidate loci in the starch metabolism pathway in Sorghum: prospects for linkage disequilibrium mapping. Crop Sci 47:S125-S134. doi:10.2135/cropsci2007.01.0054tpg

Han XZ, Campanella OH, Guan H, Keeling PL, Hamaker BR (2002a) Influence of maize starch granule-associated protein on the rheological properties of starch pastes-part II. Dynamic measurements of viscoelastic properties of starch pastes. Carbohydr Polym 49:323-330. doi:10.1016/S01448617(01)00348-4

Han XZ, Campanella OH, Guan H, Keeling PL, Hamaker BR (2002b) Influence of maize starch granule-associated protein on the rheological properties of starch pastes. Part I. Large deformation measurements of paste properties. Carbohydr Polym 49:315-321. doi:10.1016/S0144-8617(01) 00347-2

Hylton CM, Denyer K, Keeling PL, Chang MT, Smith AM (1996) The effect of waxy mutations on the granule-bound starch synthases of barley and maize endosperms. Planta 198:230-237. doi:10.1007/BF00206248

Isshiki M, Morino K, Nakajima M, Okagaki RJ, Wessler SR, Izawa T, Shimamoto K (1998) A naturally occurring functional allele of the rice waxy locus has a GT to TT mutation at the $5^{\prime}$ splice site of the first intron. Plant $\mathrm{J} 15$ : 133-138. doi:10.1046/j.1365-313X.1998.00189.x

James MG, Denyer K, Myers AM (2003) Starch synthesis in the cereal endosperm. Curr Opin Plant Biol 6:215-222. doi:10.1016/S1369-5266(03)00042-6

Jane J, Chen YY, Lee LF, McPherson AE, Wong KS, Radosavljevic M, Kasemsuwan T (1999) Effects of amylopectin branch chain length and amylose content on the gelatinization and pasting properties of starch. Cereal Chem 76: 629-637. doi:10.1094/CCHEM.1999.76.5.629

Kang HJ, Hwang IK, Kim KS, Choi HC (2006) Comparison of the physicochemical properties and ultrastructure of japonica and indica rice grains. J Agric Food Chem 54: 4833-4838. doi:10.1021/jf060221+

Kopp J, Schwede T (2004) The SWISS-MODEL repository of annotated three-dimensional protein structure homology models. Nucleic Acids Res 32:D230-D234. doi:10.1093/nar/ gkh008

Kopp J, Schwede T (2006) The SWISS-MODEL repository: new features and functionalities. Nucleic Acids Res 34: D315-D318. doi:10.1093/nar/gkj056

Larkin PD, Park WD (1999) Transcript accumulation and utilization of alternate and non-consensus splice sites in rice granule-bound starch synthase are temperature-sensitive and controlled by a single-nucleotide polymorphism. Plant Mol Biol 40:719-727. doi:10.1023/A:1006298608 408
Liu YG, Chen Y (2007) High-efficiency thermal asymmetric interlaced PCR for amplification of unknown flanking sequences. Biotechniques 43:649. doi:10.2144/000112601

McIntyre CL, Drenth J, Gonzalez N, Henzell RG, Jordan DR (2008) Molecular characterization of the waxy locus in sorghum. Genome 51:524-533. doi:10.1139/G08-035

Nakamura T, Yamamori M, Hirano H, Hidaka S, Nagamine T (1995) Production of waxy (amylose-free) wheats. Mol Gen Genet 248:253-259. doi:10.1007/BF02191591

Pedersen JF, Bean SR, Graybosch RA, Park SH, Tilley M (2005) Characterization of waxy grain sorghum lines in relation to granule-bound starch synthase. Euphytica 144: 151-156. doi:10.1007/s10681-005-5298-5

Pedersen JF, Graybosch RA, Funnell DL (2007) Occurrence of the waxy alleles wxa and wxb in waxy sorghum plant introductions and their effect on starch thermal properties. Crop Sci 47:1927-1933. doi:10.2135/cropsci2006.10.0652

Peitsch MC (1996) ProMod and Swiss-Model: internet-based tools for automated comparative protein modelling. Biochem Soc Trans 24:274-279

Ral JP, Colleoni C, Wattebled F, Dauville D, Nempont C, Deschamps P, Li Z, Morell MK, Chibbar R, Purton S, D'Hulst C, Ball SG (2006) Circadian clock regulation of starch metabolism establishes GBSSI as a major contributor to amylopectin synthesis in Chlamydomonas reinhardtii. Plant Physiol 142:305-317. doi:10.1104/pp.106.081885

Rooney LW, Pflugfelder RL (1986) Factors affecting starch digestibility with special emphasis on sorghum and corn. J Anim Sci 63:1607-1623

Rooney WL, Aydin S, Kuhlman LC (2005) Assessing the relationship between endosperm type and grain yield potential in sorghum (Sorghum bicolor L. Moench). Field Crops Res 91:199-205. doi:10.1016/j.fcr.2004.07.011

Sang Y, Bean S, Seib PA, Pedersen J, Shi YC (2008) Structure and functional properties of sorghum starches differing in amylose content. J Agric Food Chem 56:6680-6685. doi: $10.1021 / \mathrm{jf} 800577 \mathrm{x}$

Schwede T, Kopp J, Guex N, Peitsch MC (2003) SWISSMODEL: an automated protein homology-modeling server. Nucleic Acids Res 31:3381-3385. doi:10.1093/nar/gkg520

Sharma V, Rausch KD, Tumbleson ME, Singh V (2007) Comparison between granular starch hydrolyzing enzyme and conventional enzymes for ethanol production from maize starch with different amylose: amylopectin ratios. StarchStarke 59:549-556. doi:10.1002/star.200700631

Sheng F, Jia X, Yep A, Preiss J, Geiger JH (2009). The crystal structures of the open and catalytically competent closed conformation of Escherichia coli glycogen synthase. J Biol Chem. doi:10.1074/jbc.M809804200

Shure M, Wessler S, Fedoroff N (1983) Molecular identification and isolation of the waxy locus in maize. Cell 35: 225-233. doi:10.1016/0092-8674(83)90225-8

Taira T, Fujita N, Takaoka K, Uematsu M, Wadano A, Kozaki S, Okabe S (1995) Variation in the primary structure of waxy proteins (granule-bound starch synthase) in diploid cereals. Biochem Genet 33:269-281. doi:10.1007/BF02 401857

Tenorio G, Orea A, Romero JM, Merida A (2003) Oscillation of mRNA level and activity of granule-bound starch synthase I in Arabidopsis leaves during the day/night 
cycle. Plant Mol Biol 51:949-958. doi:10.1023/A:10230 53420632

Tsai CY (1974) The function of the waxy locus in starch synthesis in maize endosperm. Biochem Genet 11:83-96. doi:10.1007/BF00485766

Wang Z-Y, Zheng F-Q, Shen G-Z, Gao J-P, Snustad DP, Li MG, Zhang J-L, Hong M-M (1995) The amylose content in rice endosperm is related to the post-transcriptional regulation of the waxy gene. Plant J 7:613-622. doi:10.1046/ j.1365-313X.1995.7040613.x

Wang D, Bean S, McLaren J, Seib P, Madl R, Tuinstra M, Shi Y, Lenz M, Wu X, Zhao R (2008) Grain sorghum is a viable feedstock for ethanol production. J Ind Microbiol Biotechnol 35:313-320. doi:10.1007/s10295-008-0313-1
Wong JH, Lau T, Cai N, Singh J, Pedersen JF, Vensel WH, Hurkman WJ, Wilson JD, Lemaux PG, Buchanan BB (2009) Digestibility of protein and starch from sorghum (Sorghum bicolor) is linked to biochemical and structural features of grain endosperm. J Cereal Sci 49:73-82. doi: 10.1016/j.jcs.2008.07.013

Yanagisawa T, Kiribuchi-Otobe C, Yoshida H (2001) An alanine to threonine change in the $\mathrm{Wx}-\mathrm{D} 1$ protein reduces GBSS I activity in waxy mutant wheat. Euphytica 121: 209-214. doi:10.1023/A:1012051705517

Zeeman SC, Smith SM, Smith AM (2007) The diurnal metabolism of leaf starch. Biochem J 401:13-28. doi:10.1042/ BJ20061393 